\title{
Risk Factors and Nursing Interventions for Ventilator-Associated Pneumonia in Pediatric Intensive Care Unit at Assiut University Children Hospital
}

\author{
Asmaa Mohammad Abo El-Ella, Zienab Mohammad Mohey El-Deen \& Asmaa Abd El-Aziz Mohammad. \\ Nursing Specialist at the Technical Health Institute in Assiut, Egypt. \\ Professor of Pediatrics, Faculty of Medicine, Assiut University, Egypt. \\ Assistant Professor of Pediatric Nursing, Faculty of Nursing, Assiut University, Egypt.
}

\begin{abstract}
Ventilator-associated pneumonia (VAP) is the second most common hospital-acquired infection in pediatric intensive care units (PICUs). It is linked to increased morbidity, mortality, and length of stay in the hospital and intensive care unit, adding tremendously to health care costs. Therefore prevention is the most appropriate intervention. The aim of this study is to (1) assess nursing interventions, (2) identify the risk factors for VAP in PICU at Assiut University Children Hospital. Subjects and Method: A prospective cohort study design was utilized during one year. The study included 65 pediatric patients were divided into two groups: group (I) pediatric patients developed VAP (20 cases) and group (II) pediatric patients who did not develop VAP (45 cases). Two tools were used, (1) questionnaire sheet and (2) observation checklist. Results: It was found that the risk factors associated with VAP were duration of mechanical ventilation and inadequate application of infection control policies and care for it. Conclusion and Recommendations: There were risk factors associated with VAP were duration of mechanical ventilation and inadequate application of infection control measures and hygienic care, which increase morbidity, mortality and hence the cost of VAP in the PICU.
\end{abstract}

Keywords: Ventilator, Associated Pneumonia (VAP), Risk Factors \& Nursing Interventions.

\section{Introduction}

Nosocomial infections (NI) are an important public health problem associated with substantial morbidity and mortality in high-risk units, prolonged hospital stays, and increased health care costs. The risk of NI in pediatric patients depends not only on their age, primary disease and associated comorbidities but also, on the invasive procedures commonly used in high-risk units. However, the hospital-acquired infections occur in approximately $12 \%$ of pediatric intensive care unit children (Mireya et al., 2007, Stover et al., 2001 \& Cooper \& Haut, 2013).

In intensive care setting, mechanical ventilation is the cornerstone for the management of critically ill children. It has its own complications and hazards. One such complication is the chance of developing pneumonia termed the VAP. It is defined as pneumonia occurring after the patient has been on ventilator for more than 48 hours (Hamid et al., 2012 \& Foglia et al., 2007). VAP is an important problem in PICUs. Its prevalence ranges from 3\% to $67 \%$ in North America (Gauvin et al., 2003). It is not only associated with increased mortality but also increases with the length of ICU stay while the cost of treatment and the chances of ventilator dependence (Hamid et al., 2012).

The onset of VAP can be divided into 2 types: early and late. Early onset VAP which was developed in the first 5 to 7 days of mechanical ventilation, and late onset (was developed after 5 to 7 days of mechanical ventilation). VAP can develop at any time during ventilation, but occurs more often in the first few days after intubation. This is because the intubation process itself contributes to the development of VAP (Augustyn, 2007 \& Wikipedia, 2014).

The risk factors responsible for VAP occurrence can be classified into: host related, device related and personnel related. Host related factors include associated co-morbidities such as acute respiratory distress syndrome, cardiovascular system diseases, and central nervous system diseases; Multiple Organ System Failure (MOSF); level of consciousness; patients' body positioning; number of intubations; and medications including sedatives and prior antibiotic administration. Device-related factors include the endotracheal tube, the ventilation circuit and the presence of a nasogastric or an orogastric tube. Personnel related factors include improper hand hygiene and inadequate use of personal protective equipment by the nursing staff, resulting in crosscontamination between patients (Galal et al., 2016). Nursing interventions for prevention of VAP included oral care every 2 hours, patient repositioning every 2 hours, elevation of head of bed, suctioning method (open vs. closed), and suctioning every 2 hours. Respiratory interventions were cuff pressure checks every 2 hours, ventilator circuit changes, and ventilator condensation removal. Moreover, the important measures for prevention of VAP include

Vol , (5) No, (11) August 2017 
implementation of hand washing, reduction of duration of mechanical ventilation as far as possible, maintenance of semi-recumbent position and avoidance of the modifiable risk factors such as tracheostomy, re-intubation, corticosteroid therapy, stress ulcer prophylaxis and contaminated respiratory equipment (Curley et al., 2006 \& Joseph et al., 2010).

\section{Significance of the Study}

Ventilator-associated pneumonia is one of the most common and life-threatening problems among patients requiring artificial ventilation. Patients with VAP present a high mortality rate. So this study was conducted to identify the risk factors which lead to ventilator-associated pneumonia and to observe the nurses' interventions for those patients.

\section{Aim of the Study}

The aim of this study is to (1) assess nursing interventions, (2) identify the risk factors for VAP in PICU at Assiut University Children Hospital.

\section{Subjects \& Method \\ Research Design:}

A prospective cohort study design was utilized to meet the aim of the study.

\section{Setting}

The study was conducted at the Pediatric Intensive Care Unit, Assiut University Children Hospital.

\section{Subjects}

A convenient sample of mechanically ventilated pediatric patients who were admitted to pediatric intensive care unit aged from 31 days to 18 years, of both gender were included in the present study during the period from January 1, 2012 to December 31, 2012. The pediatric patients were classified into two groups:

- Group (I): Pediatric patients who was developed ventilator-associated pneumonia included $(n=20)$ diagnosed according to Sevketoglu and Karabocuoglu (2007) and Morinec et al (2010).

- Group (II): Pediatric patients who did not develop pneumonia on ventilator $(n=45)$.

\section{- Inclusion Criteria}

- The pediatric patients connected to mechanical ventilator for at least 48 hours or more were included.

- Exclusion Criteria

- Pediatric patients with:

- Suspected immunological disorders.

- A known underlying lung infection since admission.

- The pediatric patient who were mechanically ventilated for less than 48 hours.
Tools

The tools of the study were utilized for data collection included the following:

Tool I: Questionnaire Sheet

Questionnaire sheet was constructed after reviewing the relevant literature. It consists of three parts:

- Part I: Demographic data of the pediatric patient such as: patient name, date of admission, age, and sex.

- Part II: Medical history of the present illness such as: primary diagnosis, cause of connection to mechanical ventilation, mode of mechanical ventilation and diagnosis of ventilator-associated pneumonia.

- Part III: Ventilator-associated pneumonia, patient's risk factors such as: Drugs (sedation, paralytic medication, GI ulcer prophylaxis, deep venous thrombosis prophylaxis) presence of NGT, GI feedings, Glasgow coma scale score and duration of mechanical ventilation by days.

Tool II: Observation Checklist for Assessment of Nursing Interventions related to VentilatorAssociated Pneumonia

This tool was constructed by the researcher after reviewing related literature to assess to observe the nurses' interventions for pediatric patients connected to the mechanical ventilation as hand washing, oral care, pediatric patient repositioning, elevation of the head of bed, suctioning every 2 hours, positioning, administration of drugs (sedation, paralytic medication, GI ulcer prophylaxis, deep venous thrombosis prophylaxis), and avoidance of the modifiable risk factors such as tracheostomy, reintubation and ventilator circuit change. Tools were developed by the researcher and were tested for its content validity by experts (5) in pediatrics and pediatric Nursing with content validity index was 0.8 , internal consistency of reliability was done $r=0.83$.

\section{Methods}

- Administrative design

- An official permission was obtained from the Faculty of Nursing and delivered to the director of Assiut University Children Hospital to collect the necessary data for the study.

- Pilot Study:

- A pilot study was carried out on pediatric patients, throughout a period of 37 days, who developed and not developed ventilator-associated pneumonia in a selected setting to evaluate the applicability and clarity of the study tools. The necessary modification was done. The pediatric patients of the pilot study were included in the main study.

- Field of the Work:

The data were collected after doctor's order to connecting the pediatric patients to mechanical 
ventilator, whose chest was free from pneumonia at connection, for at least 48 hours or more to find which cases developed or not developed VAP. Data were collected from pediatric intensive care unit at Assiut University Children Hospital during the period from January 1, 2012 to December 31, 2012. The tools were filled through questionnaire. The data were collected from the pediatric patient sheet. The observation sheet was carried out while the nurses were on duty during morning and afternoon shifts every day. Nurses were observed while they were performing nursing interventions for their pediatric patients by using the direct observation technique.

\section{- Confidentiality}

Confidentiality of the research was asserted and the obtained data were available only to the researcher.

\section{- Ethical Considerations}

Research proposal was approved by the research ethics committee of faculty of Nursing, Assiut University. There is no risk for study subject during application of the research. The study will follow common ethical principles in clinical research. Confidentiality and anonymity was assured. Study subjects' privacy was considered during collection of data.

\section{Statistical Design}

The collected data were tabulated and statistically analyzed to evaluate the difference between the groups under study as regards the various variables. The statistical analysis was done using computer program SPSS ver.19. Descriptive statistics (number, percentage, mean \pm S.D and median) were done. Chisquare test was used to compare between qualitative variables. P. value was considered to be significant if $<0.05$.

\section{Results}

Table (1): Comparison between both groups of the studied pediatric patients regarding demographic characteristics.

\begin{tabular}{|c|c|c|c|c|c|c|}
\hline \multirow[t]{2}{*}{ Demographic Data } & \multicolumn{2}{|c|}{$\begin{array}{l}\text { Group (I) } \\
(n=20)\end{array}$} & \multicolumn{2}{|c|}{$\begin{array}{l}\text { Group (II) } \\
(n=45)\end{array}$} & \multirow[t]{2}{*}{$\mathbf{X}^{2}$} & \multirow[t]{2}{*}{ P-value } \\
\hline & No. & $\%$ & No. & $\%$ & & \\
\hline \multicolumn{5}{|l|}{ Age } & \multirow{3}{*}{2.75} & \multirow{3}{*}{0.097} \\
\hline$-<3$ years & 11 & 55.0 & 34 & 75.6 & & \\
\hline - $\quad \geq 3$ years & 9 & 45.0 & 11 & 24.4 & & \\
\hline \multicolumn{5}{|l|}{ Sex } & \multirow{3}{*}{0.02} & \multirow{3}{*}{0.896} \\
\hline - $\quad$ Male & 13 & 65.0 & 30 & 66.7 & & \\
\hline - $\quad$ Female & 7 & 35.0 & 15 & 33.3 & & \\
\hline
\end{tabular}

Table (2): Comparison between both groups of the studied pediatric patients regarding clinical criteria of ventilator-associated pneumonia.

\begin{tabular}{|c|c|c|c|c|c|c|}
\hline \multirow{2}{*}{ Clinical Criteria } & \multicolumn{2}{|c|}{$\begin{array}{c}\text { Group (I) } \\
(\mathbf{n}=\mathbf{2 0})\end{array}$} & \multicolumn{2}{|c|}{$\begin{array}{c}\text { Group (II) } \\
(n=45)\end{array}$} & \multirow{2}{*}{$\mathbf{X}^{2}$} & \multirow{2}{*}{ P-value } \\
\hline & No. & $\%$ & No. & $\%$ & & \\
\hline \multicolumn{5}{|l|}{ Fever } & \multirow{3}{*}{49.35} & \multirow{3}{*}{$0.000 *$} \\
\hline - Present & 20 & 100.0 & 4 & 8.9 & & \\
\hline - $\quad$ Not present & 0 & 0.0 & 41 & 91.1 & & \\
\hline \multicolumn{5}{|l|}{ Leukocytosis } & \multirow{3}{*}{35.86} & \multirow{3}{*}{$0.000 *$} \\
\hline - $\quad$ Present & 20 & 100.0 & 9 & 20.0 & & \\
\hline - $\quad$ Not present & 0 & 0.0 & 36 & 80.0 & & \\
\hline \multicolumn{5}{|l|}{ Purulent secretions } & \multirow{3}{*}{49.35} & \multirow{3}{*}{$0.000 *$} \\
\hline - $\quad$ Present & 20 & 100.0 & 4 & 8.9 & & \\
\hline - $\quad$ Not present & 0 & 0.0 & 41 & 91.1 & & \\
\hline \multicolumn{5}{|l|}{ Tachypnea } & \multirow{3}{*}{12.52} & \multirow{3}{*}{$0.000 *$} \\
\hline - $\quad$ Present & 13 & 65.0 & 9 & 20.0 & & \\
\hline - $\quad$ Not present & 7 & 35.0 & 36 & 80.0 & & \\
\hline
\end{tabular}




\begin{tabular}{|c|c|c|c|c|c|c|}
\hline \multirow{2}{*}{ Clinical Criteria } & \multicolumn{2}{|c|}{$\begin{array}{c}\text { Group (I) } \\
(\mathbf{n}=\mathbf{2 0}) \\
\end{array}$} & \multicolumn{2}{|c|}{$\begin{array}{c}\text { Group (II) } \\
(n=45)\end{array}$} & \multirow{2}{*}{$\mathbf{X}^{2}$} & \multirow{2}{*}{ P-value } \\
\hline & No. & $\%$ & No. & $\%$ & & \\
\hline \multicolumn{5}{|l|}{ Crackles breath sounds } & \multirow{3}{*}{2.77} & \multirow{3}{*}{0.096} \\
\hline - $\quad$ Present & 12 & 60.0 & 17 & 37.8 & & \\
\hline - $\quad$ Not present & 8 & 40.0 & 28 & 62.2 & & \\
\hline \multicolumn{5}{|l|}{ Worsening Gas Exchange } & \multirow{3}{*}{22.57} & \multirow{3}{*}{$0.000 *$} \\
\hline - $\quad$ Present & 13 & 65.0 & 4 & 8.9 & & \\
\hline$-\quad$ Not present & 7 & 35.0 & 41 & 91.1 & & \\
\hline
\end{tabular}

* Significant $(p<0.05)$

Table (3): Comparison between both groups of the studied pediatric patients regarding radiological criteria of ventilator-associated pneumonia.

\begin{tabular}{|c|c|c|c|c|c|c|}
\hline \multirow[t]{2}{*}{ Radiological Criteria } & \multicolumn{2}{|c|}{$\begin{array}{c}\text { Group (I) } \\
(\mathbf{n}=\mathbf{2 0})\end{array}$} & \multicolumn{2}{|c|}{$\begin{array}{c}\text { Group (II) } \\
(n=45)\end{array}$} & \multirow[t]{2}{*}{$\mathbf{X}^{2}$} & \multirow[t]{2}{*}{ P-value } \\
\hline & No. & $\%$ & No. & $\%$ & & \\
\hline \multicolumn{5}{|c|}{ Progressive pulmonary infiltrates } & \multirow{3}{*}{65.00} & \multirow{3}{*}{$0.000 *$} \\
\hline Present & 20 & 100.0 & 0 & 0.0 & & \\
\hline$-\quad$ Not present & 0 & 0.0 & 45 & 100.0 & & \\
\hline \multicolumn{5}{|l|}{ Cavitations } & \multirow{3}{*}{5.20} & \multirow{3}{*}{$0.023^{*}$} \\
\hline - $\quad$ Present & 15 & 75.0 & 20 & 44.4 & & \\
\hline - $\quad$ Not present & 5 & 25.0 & 25 & 55.6 & & \\
\hline \multicolumn{5}{|l|}{ Air bronchograms } & \multirow{3}{*}{4.13} & \multirow{3}{*}{$0.042 *$} \\
\hline - $\quad$ Present & 13 & 65.0 & 17 & 37.8 & & \\
\hline$-\quad$ Not present & 7 & 35.0 & 28 & 62.2 & & \\
\hline
\end{tabular}

* Significant $(p<0.05)$

Table (4): Comparison of patients' risk factors among the two groups of the studied cases

\begin{tabular}{|c|c|c|c|c|c|c|}
\hline \multirow{2}{*}{$\begin{array}{c}\text { Pediatric patient's Risk } \\
\text { Factors }\end{array}$} & \multicolumn{2}{|c|}{$\begin{array}{c}\text { Group }(I) \\
(n=20)\end{array}$} & \multicolumn{2}{|c|}{$\begin{array}{c}\text { Group (II) } \\
\quad(n=45)\end{array}$} & \multirow[t]{2}{*}{$\mathbf{X}^{2}$} & \multirow[t]{2}{*}{ P-value } \\
\hline & No. & $\%$ & No. & $\%$ & & \\
\hline \multicolumn{5}{|l|}{ Sedation } & \multirow{3}{*}{2.16} & \multirow{3}{*}{0.142} \\
\hline - $\quad$ Given & 11 & 55.0 & 16 & 35.6 & & \\
\hline - $\quad$ Not given & 9 & 45.0 & 29 & 64.4 & & \\
\hline \multicolumn{5}{|l|}{ Paralytic medication } & \multirow{3}{*}{2.17} & \multirow{3}{*}{0.332} \\
\hline - $\quad$ Given & 3 & 15.0 & 2 & 4.4 & & \\
\hline - $\quad$ Not given & 17 & 85.0 & 43 & 95.6 & & \\
\hline \multicolumn{5}{|l|}{ Nasogastric tube } & \multirow{3}{*}{0.15} & \multirow{3}{*}{0.941} \\
\hline - $\quad$ Present & 16 & 32.0 & 34 & 68.0 & & \\
\hline Not present & 4 & 26.7 & 11 & 73.3 & & \\
\hline \multicolumn{5}{|l|}{ Gastrointestinal feedings } & \multirow{3}{*}{0.01} & \multirow{3}{*}{0.934} \\
\hline - $\quad$ Present & 10 & 50.0 & 22 & 48.9 & & \\
\hline - $\quad$ Not present & 10 & 50.0 & 23 & 51.1 & & \\
\hline \multicolumn{5}{|c|}{ Gastrointestinal ulcer prophylaxis } & \multirow{3}{*}{1.62} & \multirow{3}{*}{0.203} \\
\hline - $\quad$ Given & 12 & 60.0 & 34 & 75.6 & & \\
\hline - $\quad$ Not given & 8 & 40.0 & 11 & 24.4 & & \\
\hline
\end{tabular}


Table (5): Comparison between both groups of all studied pediatric patients regarding outcome.

\begin{tabular}{|c|c|c|c|c|c|c|}
\hline \multirow[t]{2}{*}{ Outcome } & \multicolumn{2}{|c|}{$\begin{array}{c}\text { Group (I) } \\
(\mathbf{n}=\mathbf{2 0})\end{array}$} & \multicolumn{2}{|c|}{$\begin{array}{c}\text { Group (II) } \\
(n=45)\end{array}$} & \multirow[t]{2}{*}{$\mathbf{X}^{2}$} & \multirow{2}{*}{$\begin{array}{c}\text { P- } \\
\text { value }\end{array}$} \\
\hline & No. & $\%$ & No. & $\%$ & & \\
\hline Recovered and transmitted to pediatric intermediate ICU & 5 & 25.0 & 9 & 20.0 & \multirow{2}{*}{0.21} & \multirow{2}{*}{0.900} \\
\hline Died & 15 & 75.0 & 36 & 80.0 & & \\
\hline
\end{tabular}

Table (6): Comparison between the duration of mechanical ventilation days among the two studied groups.

\begin{tabular}{|c|c|c|c|c|c|c|}
\hline \multirow{2}{*}{$\begin{array}{c}\text { Duration of mechanical ventilation } \\
\text { days }\end{array}$} & \multicolumn{2}{|c|}{$\begin{array}{c}\text { Group }(I) \\
(n=20)\end{array}$} & \multicolumn{2}{|c|}{$\begin{array}{l}\text { Group (II) } \\
(n=45)\end{array}$} & \multirow[t]{2}{*}{$\mathbf{X}^{2}$} & \multirow[t]{2}{*}{ P-value } \\
\hline & No. & $\%$ & No. & $\%$ & & \\
\hline$<10$ days & 5 & 25.0 & 30 & 66.7 & \multirow{3}{*}{10.30} & \multirow{3}{*}{$0.006^{*}$} \\
\hline $10-<20$ days & 6 & 30.0 & 8 & 17.8 & & \\
\hline$\geq 20$ days & 9 & 45.0 & 7 & 15.6 & & \\
\hline
\end{tabular}

* Significant $(p<0.05)$

Table (7): Percentage distribution of interventions for ventilator-associated pneumonia of all studied pediatric patients.

\begin{tabular}{|l|c|c|c|c|c|c|}
\hline \multirow{2}{*}{ Items } & \multicolumn{2}{|c|}{ Done Complete } & \multicolumn{2}{c|}{ Done Incomplete } & \multicolumn{2}{c|}{$\begin{array}{c}\text { Not } \\
\text { Done }\end{array}$} \\
\cline { 2 - 7 } & No. & $\%$ & No. & $\%$ & No. & $\%$ \\
\hline$\bullet$ Hand washing & 23 & 35.4 & 32 & 49.2 & 10 & 15.4 \\
\hline$\bullet$ Oral care & 13 & 20.0 & 19 & 29.2 & 33 & 50.8 \\
\hline$\bullet$ Patient repositioning & 55 & 84.6 & 0.0 & 0.0 & 10 & 15.4 \\
\hline$\bullet$ Elevation of head of bed & 58 & 89.2 & 0.0 & 0.0 & 7 & 10.8 \\
\hline$\bullet$ Suctioning every 2 hours & 58 & 89.2 & 7 & 10.8 & 0.0 & 0.0 \\
\hline$\bullet$ Maintenance of semi-recumbent position & 34 & 52.3 & 0.0 & 0.0 & 31 & 47.7 \\
\hline$\bullet$ Deep venous thrombosis prophylaxis & 4 & 6.2 & 0.0 & 0.0 & 61 & 93.8 \\
\hline$\bullet$ Peptic ulcer disease prophylaxis & 46 & 70.8 & 0.0 & 0.0 & 19 & 29.2 \\
\hline$\bullet$ Modifiable risk factors such as: & & & & & & \\
\hline - Re-intubation & 9 & 13.8 & 0.0 & 0.0 & 56 & 86.2 \\
\hline - Ventilator circuit change & 43 & 66.2 & 0.0 & 0.0 & 22 & 33.8 \\
\hline
\end{tabular}

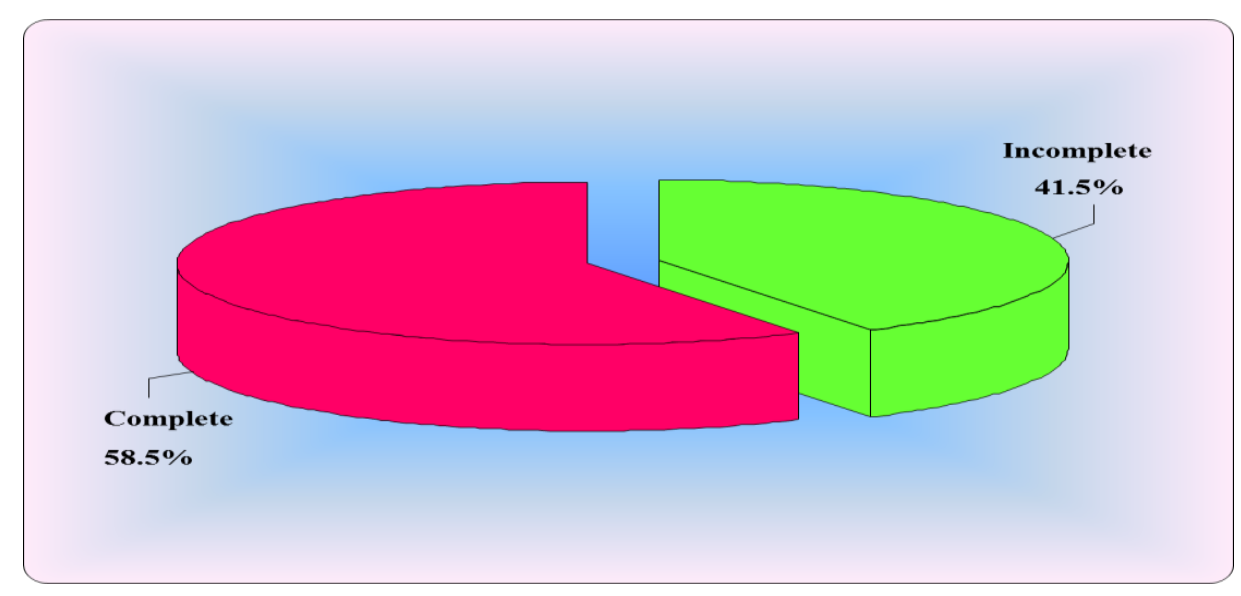

Figure (1): Frequency distribution of the total score of nurses' performance of the studied pediatric patients 


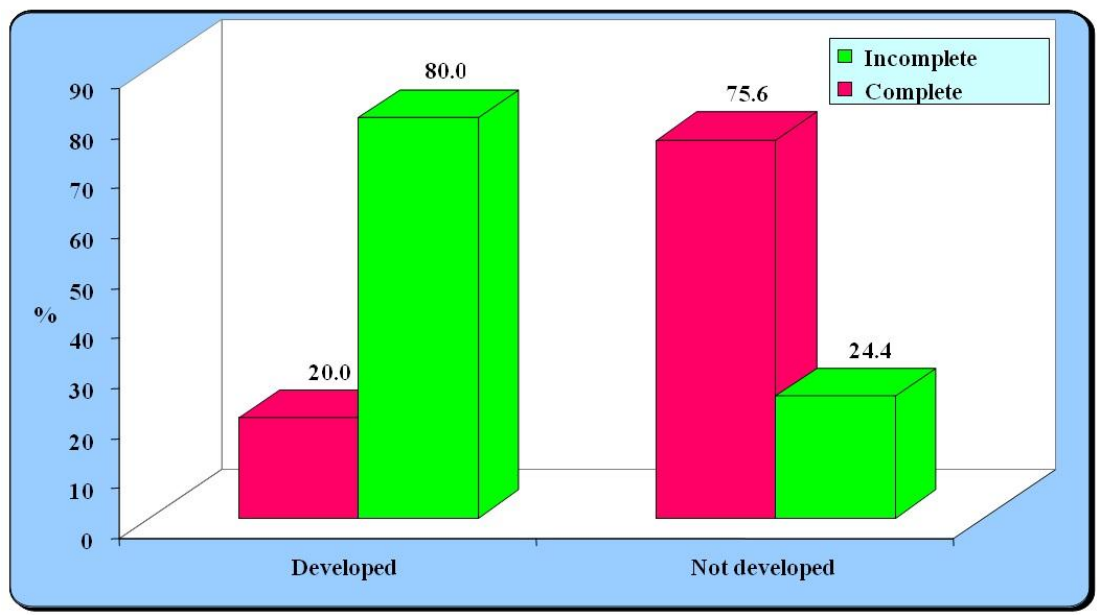

Figure (2): Relation between nurses' performance and prognosis of the studied pediatric patients

Table (1): showed that the most commonly affected age group in all studied cases (developed and not developed VAP) was 31 days to $<3$ years $(55 \%)$ and male patients $(65 \%)$ more affected than female. It was found that there was no significant statistical difference between two groups regarding demographic characteristics.

Table (2): showed the relationship between both groups of all studied cases regarding clinical criteria. There was significant statistical difference between the two groups regarding clinical criteria (p. 0.000) except crackles breath sounds.

Table (3): showed the relationship between both groups of all studied cases regarding radiological criteria. There was significant statistical difference between both groups regarding radiological criteria.

Table (4): showed the relationship between both groups of all studied cases regarding pediatric patient's risk factors. There was no significant statistical difference between two groups regarding pediatric patient's risk factors.

Table (5): showed that $20(30.8 \%)$ of cases fit the VAP criteria and more than two thirds of cases $(75$ $\%)$ who developed pneumonia died while 45 (69.2 $\%$ ) of cases not fit the VAP criteria and $80 \%$ of them died and there was no significant statistical difference between two groups regarding outcome.

Table (6): showed that pediatric patients who didn't develop ventilator-associated pneumonia had significantly shorter duration of connection to mechanical ventilation in comparison to those who developed ventilator-associated pneumonia (p. 0.006).

Table (7): showed percentage distribution of interventions for ventilator-associated pneumonia of all studied cases, more than two fifths of medical team $(49.2 \%)$ washed their hands in an incomplete manner when providing medical and nursing care to the patients. Half of all cases $(50.8 \%)$ didn't receive oral care. The majority of cases had received repositioning care $(84.6 \%)$, elevated the head of bed $(89.2 \%)$ and suctioning every two hours $(89.2 \%)$ completely. More than half of cases $(52.3 \%)$ had maintenance of semi-recumbent position. The majority of cases didn't receive deep venous thrombosis prophylaxis $(93.8 \%)$ and more than two thirds of cases $(70.8 \%)$ received peptic ulcer disease prophylaxis. The modifiable risk factors such as: reintubation didn't done for $(86.2 \%)$ of cases and ventilator circuit was changed for $(66.2 \%)$ of all cases.

Figure (1): showed that $(58.5 \%)$ of nurses' performance was satisfactory, while $(41.5 \%)$ of their performance was unsatisfactory.

Figure (2): showed the relation between nurses' performance and prognosis of all studied cases. There was significant statistical difference between nurses' performance and prognosis of all studied cases.

\section{Discussion}

VAP is an important problem in PICUs. VAP is defined as pneumonia occurring after the patient has been on ventilator for more than 48 hours. VAP is different from community acquired pneumonia not only from etiological point of view but also in context of its pathophysiology, risk factors, management strategies and outcome. VAP is a marked health risk for hospitalized infants and children. It is one of the top causes of hospitalacquired infection in the PICU (Hamid et al., 2012; Foglia et al., 2007 \& Cooper \& Haut, 2013).

Our study was done at Assiut University Children Hospital which is the only ICU in Upper Egypt and it drains 8 governorates. In this study, the cases had been diagnosed according to Morinec et al., (2010). The present study revealed that there was statistical 
significant difference between the two groups regarding clinical and radiological criteria. In the present study, VAP was detected among 20 of studied cases who fit the VAP criteria.

The present study (Table 1) found that the most commonly affected age group was 31 days to $<3$ years $(55 \%)$ and male patients $(65 \%)$ more affected than female. This finding agrees with Sevketoglu \& Karabocuoglu, (2007) who stated that the most commonly affected age group was 2 to 12 -months. In the same line, Hamid et al., (2012) mentioned that less than one year to be associated with increased chances of VAP. A study by the Preventive Pediatric Network reported the incidence in the $0-5$ years age group was twice that of the 5 to 12 year age group (Venkatachalam et al., 2011).

The present study (Table 4) revealed that there was no statistical significant difference between two groups regarding patient's risk factors. Amanullah \& Mosenifar (2013) reported that protocols for sedation and weaning should be applied in the ICU to reduce the duration of mechanical ventilation.

Decreasing use of narcotic and/or sedative agents in the intensive care unit must be done cautiously, because pain can limit deep breathing and impair oxygenation. Daily interruptions of continuous sedative infusions can shorten the duration of mechanical ventilation by more than 2 days and length of stay in the intensive care unit by 3 - 5 days (Augustyn 2007).

The present study revealed that $20(30.8 \%)$ of cases fit the VAP criteria and more than two thirds of cases (75 \%) who developed pneumonia died while 45 $(69.2 \%)$ of cases not fit the VAP criteria and $80 \%$ of them died and there was no significant statistical difference between two groups regarding outcome. (Table 5) This result may be explained by the fact that cases who admitted in the PICU had delayed arrival or unplanned admission with multiple organ failure which carries poor prognosis.

This agrees with Fathy et al., (2013) who studied the incidence/proportion of VAP in Egyptian University hospitals. The age of the patients ranged from 2 days to 77 years. Eight studies concerned with incidence of VAP in three Universities; Alexandria (four studies), Ain Shams (three studies) and Mansoura (one study). Incidence of VAP ranged from $16 \%$ to $75 \%$, with the lowest ratio $(16 \%)$ in Alexandria and the highest one $(75 \%)$ in Ain Shams University.

In the same line, Cooper \& Haut (2013) added that VAP is a marked health risk for hospitalized infants and children. It is one of the top causes of HAI in the PICU, accounting for $18 \%$ to $26 \%$ of all HAIs in the unit (in the PICU, $20 \%$ of NIs are VAP, with an incidence of 4 to 44 per 1000 intubated children.
Cooper \& Haut (2013), Casado et al., (2011) \& World Health Organization Media Centre (2012) also added that VAP result in a mortality rate of about $10 \%$ to $20 \%$. VAP is associated with increased mortality and morbidity, increased length of hospital stay, and high health care costs. Currently, pneumonia is the sixth leading cause of death in the United States and the leading cause of death of children worldwide. The mortality rate for patients of all ages with VAP is approximately $33 \%$ to $50 \%$.

This study found no significant difference between the two groups regarding outcome with the majority of pediatric patients in both groups died (table 5). This finding is in agreement with Sevketoglu \& Karabocuoglu, (2007) who reported that three recent pediatric studies have similarly demonstrated that there was no significant difference between VAP and non-VAP patients regarding attributable mortality in children.

Mansour \& Bendary, (2012) added that Twentyfive patients out of 90 admissions $(27.7 \%)$ developed HAP during the observation period, with incidence rate of 13 per 1000 patient-days and overall mortality of $56 \%$.

The present study (Table 6) revealed that patients who didn't develop ventilator-associated pneumonia had significantly shorter duration of connection to mechanical ventilation in comparison to those who developed ventilator-associated pneumonia ( $\mathrm{p}$. 0.006). This agrees with Venkatachalam et al., (2011) who mentioned that VAP is clearly associated with increased morbidity. Multiple studies have linked VAP to an increased duration of ventilation as much as 5 - 11 days and longer PICU stay by 20 - 34 days.

In the same line, Mansour \& Bendary, (2012) said in their study that the mean length of stay was doubled following HAP together with $50 \%$ increment in the mean duration of mechanical ventilation days. In another study, HAP lengthens the hospital stay by 7 - 9 days and is associated with a higher cost of medical care.

The results of the present study (Figure 1,2) revealed that there was significant statistical difference between nurses' performance regarding prognosis (0.000) of all studied cases. Augustyn, (2007) said that improper hand washing resulting in the crosscontamination of patients is the biggest personnelrelated risk factor for VAP. Patients who are intubated and receiving mechanical ventilation often need interventions such as suctioning or manipulation of the ventilator circuit. These interventions increase the likelihood of cross-contamination between patients if healthcare staff didn't use proper hand washing techniques. Failure to wash hands and change gloves between contaminated patients has 
been associated with an increased incidence of VAP. In addition, failure to wear proper personal protectresistant organisms have been identified increases the risk of cross-contamination between patients.

Augustyn (2007) mentioned that positioning patients in a semi-recumbent position with the head of the bed elevated $30^{\circ}$ to $45^{\circ}$ prevents reflux and aspiration of bacteria from the stomach into the airways. Simply elevating the head of the bed $30^{\circ}$ can decrease VAP by $34 \%$. Other host-related factors include patients' body positioning, level of consciousness, number of intubations, and medications, including sedative agents and antibiotics.

Amanullah \& Mosenifar (2013) added that patient position can be associated with an increased incidence of hospital-acquired pneumonia (HAP) and VAP. The incidence of HAP is increased in supine patients when compared with semi-recumbent patients, although there was no difference in mortality. Placing patients in a semi-recumbent position is associated with approximately a 3-fold reduction in the risk of HAP, especially during enteral0 feeding.

Augustyn, (2007) reported that maintenance of aseptic technique when performing endotracheal suctioning is essential to prevent contamination of the airways. No difference has been found in the incidence of VAP with open versus closed suction systems. When a closed system is used, the suction catheter should be rinsed free of secretions away from the patient. Routine turning of patients a minimum of every 2 hours can increase pulmonary drainage and decrease the risk for VAP.

Amanullah \& Mosenifar (2013) said that continuous aspiration of subglottic secretions reduces the risk of early-onset VAP. Results of a randomized, controlled trial showed a significant reduction in VAP (relative risk reduction of $42 \%$ ), including lateonset VAP, when subglottic secretion drainage was performed while patients were on mechanical ventilation. Cuff pressures should be maintained at greater than $20 \mathrm{~cm}$ of water to prevent aspiration around the endotracheal tube.

Wright \& Romano, (2006) reported that as part of the ventilator bundle, DVT prophylaxis for mechanically ventilated patients also is recommended as excellent practice. Clinical guidelines found in the Seventh American College of Chest Physician's Conference on Antithrombotic and Thrombolytic Therapy recommend that DVT prophylaxis be instituted in patients admitted for surgery, trauma, or acute medical illness, as well as for all patients admitted to the ICU. This practice is very reasonable and is targeted at decreasing the comorbidities associated with ICU admission and mechanical ventilation. No data specifically address the impact of
DVT prophylaxis on the incidence of VAP. Iacoboni, (2010) added that most common risk factor for VAP: DVT prophylaxis (80\% of patients did not have sequential compression devices).

Augustyn (2007) added that colonization of the ventilator circuit can also play a role in the development of VAP. Daily changes of the ventilator circuit do not seem to decrease the incidence of VAP. The Centers for Disease Control and Prevention does not recommend changing the ventilator circuit more than once every 48 hours, and research has indicated that changing the ventilator circuit as infrequently as once a week does not increase the risk for VAP. It is recommended that the ventilator circuit be changed when visibly soiled.

\section{Conclusion}

Based on the results of the present study, it could be concluded that

There were risk factors associated with VAP were duration of mechanical ventilation and inadequate application of infection control measures and hygienic care, which increase morbidity, mortality and hence the cost of VAP in the PICU. This is due to the extreme shortage of nurses and lack of facilities and supplies and lack of nurses training in the intensive care unit and also other health team not always follows infection control standards. So Bundle to prevent VAP in children which include hand washing, oral care, patient repositioning, elevation of head of bed, suctioning every 2 hours, maintenance of semi-recumbent position and avoidance of modifiable risk factors such as: reintubation and ventilator circuit change must be understand and accurately applied

\section{Recommendations}

Based on the finding of this study, the following recommendations were suggested

- Planning and implementation of a pediatric specific VAP prevention bundle should be done because it can produce sustained decreases VAP rates in PICU.

- Use of ventilator pathways and/or protocols with preprinted order sets can also lead to improved outcomes for patients.

- Try to increase number of nurses working in the PICU to overcome the shortness of nurses' number by decision maker.

- Improving facilities, supplies and equipments in the PICU to provide ideal care for the patient.

- Training programs provided by the work institution promote tailoring of the information to the needs of the nursing staff, as well as to the 
information required to meet the standards of the institution.

- Planning Arabic and English procedure book for nurses should be available in the units to be used as a guide for nursing action.

- Education on the prevention of VAP is essential, because the occurrence of nosocomial infections is directly related to the adequacy of staff behavior.

- Nurses need to understand the pathophysiology of VAP, risk factors for this type of pneumonia, and strategies that may prevent the disease.

\section{References}

1. Amanullah S., \& Mosenifar Z., (2013): Ventilator-Associated Pneumonia Overview of Nosocomial Pneumonias, WebMD LLC., http://emedicine.medscape.com/article/304836overview\#aw2aab6b5.

2. Augustyn B., (2007): Ventilator-Associated Pneumonia: Risk Factors and Prevention, The American Association of Critical-Care Nurses, ccn.aacnjournals.org/content/27/4/32.full, 27 : $32-39$.

3. Casado J., De Mello J., \& De Aragao C., (2011): Incidence and Risk Factors for Health Care-Associated Pneumonia in a Pediatric Intensive Care Unit, Crit Care Med., 39 (8), p: 1968 - 1973.

4. Cooper V., \& Haut C., (2013): Preventing Ventilator-Associated Pneumonia in Children: An Evidence-Based Protocol, American Association of Critical-Care Nurses, Crit Care Nurse, Vol. (33), No. (3), p: 21 - 29.

5. Curley M., Schwalenstocker E., Deshpande J., Ganser C., Bertoch D., \& Brandon J., (2006): Tailoring The Institute For Healthcare Improvement 100,000 Lives Campaign To Pediatric Settings: The Example Of VentilatorAssociated Pneumonia, Pediatric Clinics of North America, 53, p: 1231 - 1251.

6. Fathy A., Abd El-hafeez R., EL-Gilany A., Abd El-hafez S., (2013): Analysis of Ventilator Associated Pneumonia (VAP) Studies in Egyptian University Hospitals Egyptian, Journal of Chest Diseases and Tuberculosis, Elsevier, 62, p: $17-25$.

7. Foglia E., Meier D., \& Elward A., (2007): Ventilator-Associated Pneumonia in Neonatal and Pediatric Intensive Care Unit Patients, American Society for Microbiology, Clin Microbiol Rev, Vol. 20, No. 3, p: 409 - 25.

8. Galal Y., Youssef M., \& Ibrahiem S., (2016): Ventilator-Associated Pneumonia: Incidence, Risk Factors and Outcome in Paediatric Intensive Care Units at Cairo
University Hospital, J Clin Diagn Res., 10(6): SC06-SC11,

https://www.ncbi.nlm.nih.gov/pmc/articles/PM C4963727/.

9. Gauvin F., Dassa C., Chaïbou M., Proulx F., Farrell C., \& Lacroix J., (2003): VentilatorAssociated Pneumonia in Intubated Children: Comparison of Different Diagnostic Methods, The Society of Critical Care Medicine and the World Federation of Pediatric Intensive and Critical Care Societies, Pediatr Crit Care Med, Vol. (4), No. (4), p: 437.

10. Hamid M., Malik M., Masood J., Zia A., \& Ahmad T., (2012): Ventilator-Associated Pneumonia in Children, Journal of the College of Physicians and Surgeons Pakistan, Vol. 22 (3): $155-158$.

11. Iacoboni J., (2010): Risk Factors and Interventions for Ventilator Associated Pneumonia Among Ventilated Pediatric Intensive Care Unit Patients, Elsevier Inc., Journal of Pediatric Nursing, Vol. (25), Issue (2), p: e17, http://www.pediatricnursing.org/article/S08825963(09)00394-7/fulltext.

12. Joseph N., Sistla S., Dutta T., Badhe A., \& Parija S., (2010): Ventilator-Associated Pneumonia: A Review, European Journal of Internal Medicine, Vol. (21), Issue (5), p: 360368.

13. Mansour M., \& Bendary S., (2012): HospitalAcquired Pneumonia in Critically Ill Children: Incidence, Risk Factors, Outcome and Diagnosis with Insight on the Novel Diagnostic Technique of Multiplex Polymerase Chain Reaction, The Egyptian Journal of Medical Human Genetics, Ain Shams University, Production and hosting by Elsevier B.V., 13, 99 - 105, www.sciencedirect.com.

14. Mireya U., Marti P., Xavier K., Cristina L., Miguel M., \& Magda C., (2007): Nosocomial Infections in Pediatric and Neonatal Intensive Care Units, Elsevier Ltd. Journal of Infection www.elsevierhealth.com/journals/jinf, (54), $212 \mathrm{e} 220$.

15. Morinec J., Iacaboni J., \& McNett M., (2010): Risk Factors and Interventions for Ventilator-Associated Pneumonia in Pediatric Patients, Elsevier Inc., WwW.sciencedirect.com/science/article/pii/S088 2596310002368.

16. Sevketoglu E., \& Karabocuoglu M., (2007): Ventilator-associated pneumonia in children, childscience.org/html/jpic/JPIC.Sample.Review. Article.doc. 
17. Stover H., Shulman T., Bratcher F., Brady T., Levine L., \& Jarvis R., (2001): Nosocomial Infection Rates in US Children's Hospitals' Neonatal and Pediatric Intensive Care Units, Am J Infect Control, (29), p: 152 - 157.

18. Venkatachalam V., Hendley J., \& Willson D., (2011): The Diagnostic Dilemma of VentilatorAssociated Pneumonia in critically ill children, The Society of Critical Care Medicine and the World Federation of Pediatric Intensive and Critical Care Societies, Pediatr Crit Care Med, Vol. 12, No. (3), p: 286 - 293.

19. Wikipedia, the free encyclopedia, (2014): Ventilator-associated pneumonia, Wikimedia Foundation, Inc., http://en.wikipedia.org/wiki/ Ventilator-associated_pneumonia.

20. World Health Organization Media Centre, (2012): Pneumonia, Fact sheet 331, http://www.who.int/mediacentre/factsheets/fs33 1/en/ index.html.

21. Wright M., \& Romano M., (2006): VentilatorAssociated Pneumonia in Children, Elservier Inc., p: 58. 